\title{
Fuel Efficiency Optimization Methodologies for Series Hybrid Electric Vehicles
}

\author{
Boli Chen, Simos A. Evangelou and Roberto Lot
}

\begin{abstract}
This paper provides an overview of various optimization formulations that can lead to improved fuel economy for a series hybrid electric vehicle (HEV). The relevance and improvement to the current state-of-the-art are discussed. The formulated optimal control problems (OCP) consist of two individual optimization challenges: vehicle speed optimization and powertrain power-split optimization. These OCPs can be merged leading to a practical and global problem, where all the aspects are optimized simultaneously for a prescribed route and traveling time. Alternatively, the global problem can be approximated by solving individual OCPs, one for each aspect, in steps and combined a posteriori. The optimal solutions in each case are investigated and compared by simulation examples to expose the trade-off between optimality of fuel economy achieved by global optimization and reduction in computational complexity and hence practicality of the twostep solution approximation.
\end{abstract}

\section{INTRODUCTION}

Increasing concerns on global environmental pollution lead to the electrification of the automotive industry. The fuel economy of a hybrid electric vehicle (HEV) may be enhanced as with conventional vehicles by improving efficiency in two aspects: a) "tank-to-vehicle" efficiency (e.g. powertrain efficiency and energy recovery during braking), and b) "vehicle-to-miles" efficiency both by design (reduction of vehicle mass, aerodynamic drag and tyre friction) and more efficient driving (eco-driving). In the literature on HEV fuel efficiency optimization a) is usually formulated as an energy management problem, where the power-split of multiple energy sources is optimized to minimize overall fuel consumption. From a control perspective, b) is primarily addressed by optimizing driving speed in terms of energy efficiency. Both a) and b) are discussed in this paper as well as the advantages and difficulties of simultaneous optimization of a) and b) as compared to the conventional approach of individual optimization of these aspects. The focus of the paper is on the series HEV architecture, nevertheless the ideas that will be presented also have relevance to other architectures.

The problem of finding the optimal driving speed of a conventional vehicle in terms of fuel efficiency for a given route has been investigated in the literature. The pulse and glide $(\mathrm{PnG})$ strategy has been proved to be the most energy efficient driving strategy for car-following and free-driving scenarios [1]. The PnG includes a rapid acceleration at the

B. Chen and S. A. Evangelou are with the Dept. of Electrical and Electronic Engineering at Imperial College London, UK (boli.chen10@ic.ac.uk, s.evangelou@ic.ac.uk).

R. Lot is with the Dept. of Engineering and the Environment at the University of Southampton, UK (roberto. lotesoton.ac.uk).

This research was supported by the EPSRC Grant EP/N022262/1. beginning until the vehicle reaches its maximum speed, followed by a period of coasting to a low speed, where the pattern can be repeated. Nevertheless, the PnG may be not optimal for HEVs, which allow energy recovery by regenerative braking. The powertrain energy management (EM) control for a given vehicle speed profile is one of the main challenges that have emerged with the appearance of HEVs, which include multiple energy sources as opposed to the single source of conventional vehicles. Numerous EM strategies, from rule-based to optimization-based, have been proposed in the literature [2], [3], [4], [5], [6], [7]. Optimization-based EM strategies represent the most commonly used benchmarking methods as the optimal or suboptimal solutions are guaranteed in most cases. The control problem in this category is usually formulated as an optimal control problem (OCP), which is then solved by standard optimization techniques [8], [9], [10], [11]. One of the main drawbacks of the EM optimization methodologies is that they require a priori the knowledge of the entire drive cycle, which is practically unavailable.

The idea of simultaneous optimization of both speed and power-split is recently proposed by the authors in [12], where the overall fuel consumption is minimized for a given drive mission, specified in terms of the path and the desired traveling time. Compared to the existing energy management approaches, this joint optimization strategy removes the need of knowing a priori the driving cycle, which is practically unavailable. Also, it is expected to achieve better optimal solutions in contrast with conventional speed optimization methods because of the inclusion of the hybrid powertrain model. However, the computation complexity is increased when both aspects are jointly optimized. An in-depth discussion of the benefit and challenges of the simultaneous optimization strategy is still missing. The present study aims to fill the gap by examining the gain in terms of fuel economy and the sacrifice in terms of computational efficiency of the joint OCP.

The paper is structured as follows. Section II introduces the main problem to be studied and the possible OCPs that can be formulated. Driving speed optimization is addressed in Section III. In Section IV, energy management optimization of the series HEV is investigated. Simultaneous optimization of both aspects are addressed in Section V, and Section VI draws some concluding remarks.

\section{PRELIMINARIES AND PROBLEM STATEMENT}

The vehicle model studied in this work represents a general-purpose passenger car and consists of a series hybrid powertrain architecture as shown in Fig. 1 (details of the 


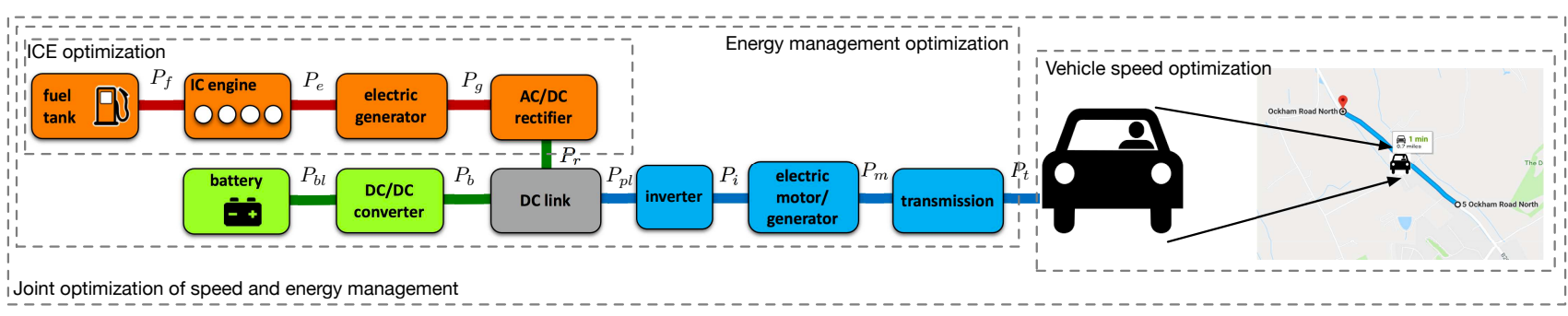

Fig. 1. Block diagram of the series HEV powertrain used in this work.

powertrain model will be introduced in Section IV-A). The main parameters of the vehicle are listed in Table I [13]. In

TABLE I

VEHICLE MAIN CHARACTERISTICS

\begin{tabular}{lcl}
\hline \hline symbol & value & description \\
\hline$m$ & $1500 \mathrm{~kg}$ & vehicle mass \\
$f_{R}$ & 0.01 & tyre rolling resistance coefficient \\
$f_{D}$ & 0.47 & aerodynamics drag coefficient \\
$Q_{\max }$ & $5 \mathrm{Ah}$ & battery capacity \\
$R_{b}$ & $0.2056 \Omega$ & battery internal resistance \\
$\eta_{r}$ & 0.96 & efficiency of the rectifier \\
$\eta_{d c}$ & 0.96 & efficiency of the DC-DC converter \\
$\eta_{i}$ & 0.96 & efficiency of the inverter \\
$\eta_{t}$ & 0.97 & efficiency of the transmission \\
$N$ & 16 & gear ratio of the transmission \\
\hline \hline
\end{tabular}

this study, the vehicle is requested to drive only for $1 \mathrm{~km}$ on a straight and flat road with specified traveling time $T$ to highlight the differences between different optimization methods, since an arbitrary path can be treated as the combination of multiple straight segments [12]. Road traffic is not considered in this work, thus the vehicle speed is not constrained by the behavior of other vehicles and infrastructure.

It is clear that the aforementioned joint optimization approach (OCP-Joint) can be adopted to find most efficient vehicle speed and power-split (between the engine and battery) jointly. Instead of OCP-Joint, the concerned problem also can be divided into two sub-problems: 1) driving speed optimization (OCP-S); 2) energy management optimization (OCP-EM), which can be solved individually in successive steps by conventional approaches and combined a posteriori, yielding a counterpart strategy (OCP-Split), which is compared to OCP-Joint in Fig. 2. In the upcoming sections,

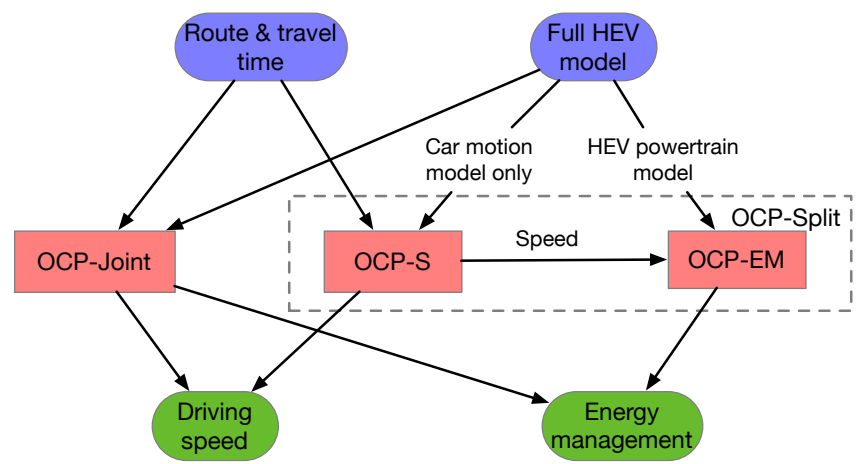

Fig. 2. Optimization problems formulated in this paper for a series HEV.

these two optimization problems, including the sub-problems will be formulated as OCPs in the following form to show the connections and to perform further comparisons:

$$
\begin{array}{cl}
\underset{\mathbf{u}}{\operatorname{minimize}} & \multicolumn{1}{c}{J(\mathbf{x}, \mathbf{u})} \\
\text { subject to: } & \frac{d}{d t} \mathbf{x}=\mathbf{f}(\mathbf{x}, \mathbf{u}, t) \\
& \psi(\mathbf{x}, \mathbf{u}, t) \leq \mathbf{0} \\
& \mathbf{b}(\mathbf{x}(0), \mathbf{x}(T))=\mathbf{0},
\end{array}
$$

where $J(\mathbf{x}, \mathbf{u})$ is given by:

$$
J(\mathbf{x}, \mathbf{u})=\int_{0}^{T} L(\mathbf{x}, \mathbf{u}, t) d t+\phi(\mathbf{x}(T))
$$

The vector $\mathbf{x}$ represents the system state vector, which evolves according to the differential equation $\dot{\mathbf{x}}=f(\mathbf{x}, \mathbf{u}, t)$, and the vector $\mathbf{u}$ represents a control input.

The OCPs formulated in this paper are solved in the Matlab environment by GPOPS-II software [14], where an OCP is approximated as sparse nonlinear programming problem (NLP), and then solved by the NLP solver IPOPT.

\section{DRIVING SPEED OPTIMIZATION (OCP-S)}

The problem of finding the energy efficient driving speed of a conventional vehicle for a given route has already been investigated in the literature. The PnG has been proved to be the optimal driving strategy for traditional vehicles in various driving conditions. In this section, the existing non-hybrid vehicle speed optimization method is extended to the hybrid vehicle case by considering energy recovery during braking, even if the precise characteristics of the power-train are not considered. The focus is instead on the driving resistance forces, which are mainly due to tire friction $F_{R}=f_{R} m g$ and aerodynamic drag resistance $F_{D}=f_{D} v^{2}$, where $m$ and $v$ are the vehicle mass and longitudinal speed respectively. The vehicle longitudinal dynamics are characterized by:

$$
m \frac{d}{d t} v=\left(F_{v}-F_{R}-F_{D}\right)
$$

where $F_{v}$ is the driving force, which is positive during traction (when the energy is transferred from the power-train to the vehicle) and negative during braking. If we define the energy recovery factor $\rho \in[0,1]$ as the ratio between regenerative braking power and the total braking power, the energy dissipation function becomes:

$$
J=\int_{0}^{T}\left\{\begin{array}{ll}
F_{v} & F_{v} \geq 0 \\
\rho F_{v} & F_{v}<0
\end{array} v d t=\int_{0}^{T} \max \left(\rho F_{v}, F_{v}\right) v d t\right.
$$


In particular, the case $\rho=0$ corresponds to a conventional power-train, while $\rho=1$ indicates that all braking energy is recovered as in a highly hybridized or fully electric vehicle. Since the speed is not known a priori, it is necessary to define the vehicle mission in terms of the traveled distance $s$, which has to be added to the system states. Finally, to ensure smooth vehicle motion and to avoid unrealistic jerky maneuvers, the vehicle is controlled in terms of the first derivative of driving force $F_{v}$. In conclusion, the state space model (1b) is:

$$
\frac{d}{d t}\left(\begin{array}{c}
s \\
v \\
F_{v}
\end{array}\right)=\left(\begin{array}{c}
v \\
\frac{1}{m}\left(F_{v}-F_{R}-F_{D}\right) \\
m j_{v}
\end{array}\right) .
$$

and has three states $\mathbf{x}=\left(s, v, F_{v}\right)$ and one input $\mathbf{u}=j_{v}$. The possible maneuvers are constrained by (1c) as follows:

$$
\begin{array}{r}
v F_{v} \leq P_{v, \max } \\
-j_{v, \max } \leq j_{v} \leq j_{v, \max } \\
v \leq v_{\max }
\end{array}
$$

where the total input power is bounded according to the individual power limits of the engine and the batter branches (introduced in later on in Section IV-A), and $j_{v}$ is bounded within $\pm 1 \mathrm{~m} / \mathrm{s}^{3}$. Moreover, the driving speed may be constrained by a legal limit $v_{\max }$.

Finally, the problem is completed by the following boundary conditions (1d):

$$
\begin{array}{cc}
s(0)=0, & s(T)=L \\
v(0)=0, & v(T)=0 \\
F_{v}(0)=0, & F_{v}(T)=0
\end{array}
$$

where $L=1 \times 10^{3} \mathrm{~m}$ is the length of the route. The vehicle is assumed static at the beginning and end of the mission for ease of comparison, thus the boundary conditions of $v$ and $F_{v}$ are set to 0 .

In the following simulation examples, the vehicle is requested to accomplish the $1 \mathrm{~km}$ mission with prescribed traveling time $T=72 \mathrm{~s}$ (i.e., average speed $50 \mathrm{~km} / \mathrm{h}$ ). The speed limit is assumed to be much higher than the average speed in the first instance, therefore the speed is not restricted by any upper limit in order to present the fully optimal driving speed in each case. In Fig. 3, the optimal speed profiles obtained by solving OCP-S for different $\rho$ are shown. As it can be noticed, when $\rho=0$, the most fuel-efficient

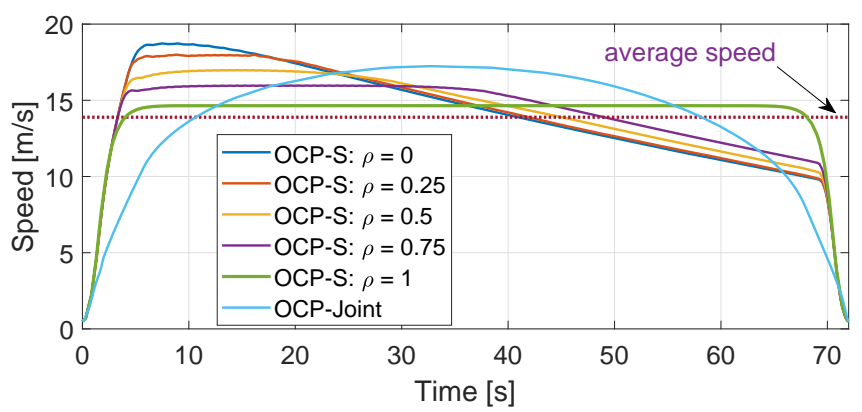

Fig. 3. Optimal speed profiles of OCP-Joint and OCP-S with different $\rho$ for a $1 \mathrm{~km}$ straight road. driving speed starts from a rapid acceleration to a maximum velocity, after that the vehicle mildly decelerates by coasting for a considerable time to a relatively low speed, at which point intensive braking is applied to stop the vehicle. Such driving behavior coincides with the PnG strategy found for conventional vehicles. However, more braking is allowed at the end of the mission as $\rho$ increases, which allows more energy to be recovered. Conversely, the peak speed at the beginning drops as $\rho$ increases to satisfy the requested average speed. When $\rho=1$, the optimal solution suggests the driver to accelerate to a cruise speed value, then to stay at this speed until the destination approaches, as drivers normally do. In conclusion, without energy recovery eco-driving requires pulse and glide, which is not natural and difficult to be implemented by drivers because the proper identification of the maximum speed point and the braking point requires a lot of anticipation. On the contrary, energy recovery does not only reduce fuel consumption, but also allows the driver to drive regularly without behavioral adaptation.

\section{Powertrain Energy Management OPTIMIZATION}

\section{A. Powertrain model}

This work uses the series powertrain model described in [12]. This dynamic model captures the essential physical characteristics of each individual component including power losses while keeping the overall model reasonably simple and appropriate for numerical optimization. As can be seen in Fig. 1, the powertrain of the vehicle comprises three branches: the propulsion load (PL), the primary source of energy (PS) and the secondary source of energy (SS). The PL is an inverter driven permanent magnet synchronous motor (PMSM), which is mechanically connected to the wheels of the car via the transmission. The PS consists of an enginegenerator unit (EGU) and a three-phase rectifier. In particular, the EGU comprises a $1.8 \mathrm{~L}$ petrol internal combustion engine (ICE), mechanically connected to a PMS generator (PMSG). The SS contains a lithium-ion battery and a bi-directional DC-DC converter. It allows energy recovery either by the PS or by regenerative braking, which conveys braking power through the transmission up to the battery. Mechanical brakes are applied directly to the wheels for deceleration, with the corresponding power converted into heat dissipation. To fulfill the desired vehicle speed and acceleration, the power from the PS, the SS, and mechanical brakes are mixed in a specified mode determined by an optimal EM strategy that is designed for fuel consumption minimization. In the following subsections, the model will be briefly introduced from one branch to another.

1) Primary source: The dynamics of the ICE (e.g., engine speed, air mass flow rate) are much faster than the dynamics of interest in the present work, such as powertrain energy flow and battery state-of-charge (SoC) variation. Therefore, it is reasonable to model the engine branch by a quasi-static modeling approach where both the engine and generator are defined in terms of an efficiency map as a function of speed and torque, while the rectifier is simply modeled as a constant efficiency factor. Since the engine, generator, and rectifier 


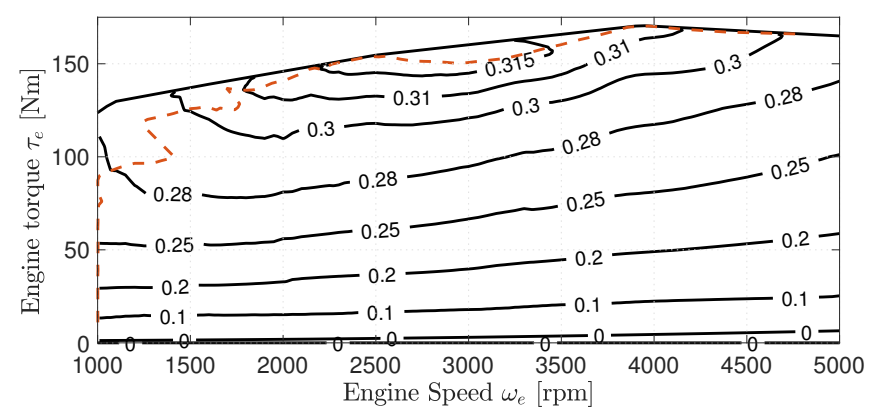

Fig. 4. Engine-Generator-Rectifier combined efficiency $\eta_{p s}$. The torquespeed operating points for maximum overall efficiency at each engine power are shown by a dashed curve.

are connected in series, the overall branch efficiency $\eta_{p s}$ is obtained by multiplying their single efficiencies and it is depicted in figure 4. Therefore, the engine fuel mass rate may be expressed as follows:

$$
\frac{d}{d t} m_{f}=\frac{1}{Q_{H V}} \frac{P_{r}}{\eta_{p s}\left(\omega_{e}, \tau_{e}\right)}
$$

where $P_{r}$ is the PS output power, $Q_{H V}$ is gasoline lower heating value, and $\omega_{e}$ and $\tau_{e}$ are engine speed and torque respectively.

2) Secondary source: The dynamic of the battery is governed by:

$$
\frac{d}{d t} \mathrm{SoC}=\frac{i_{b}}{Q_{\max }}
$$

where $i_{b}$ denotes the battery current, assumed positive during the discharge phase and $\mathrm{SoC}$ is the state-of-charge, i.e. the ratio of actual battery charge to its capacity. For a charge sustaining battery management, the battery SoC is limited within $50 \%-80 \%$ and in this range it is acceptable to assume a constant open circuit voltage: $V_{o c}=300 \mathrm{~V}$. Based on the internal resistance electrical model [15], the battery closed circuit voltage is approximated by $V_{b}=V_{o c}-R_{b} i_{b}$. Then, the battery efficiency is defined as:

$$
\eta_{b}=\left(\frac{P_{b l}}{V_{o c} i_{b}}\right)^{\operatorname{sign}\left(P_{b l}\right)}=\left(\frac{V_{o c}-R_{b} i_{b}}{V_{o c}}\right)^{\operatorname{sign}\left(P_{b l}\right)}
$$

where $P_{b l}=i_{b} V_{b}$ represents battery output power. The battery voltage is amplified by the DC/DC converter that is modeled as a constant efficiency term $\eta_{d c}$. Therefore, the bi-directional power conversion of the battery branch is described by:

$$
P_{b}=\eta_{s s}^{\operatorname{sign}\left(P_{b}\right)} P_{b l}=\eta_{s s}^{\operatorname{sign}\left(P_{b}\right)} i_{b} V_{b}
$$

where $P_{b}$ is the battery power on the DC link side, $\eta_{s s}=$ $\eta_{b} \eta_{d c}$ is the overall efficiency of the battery branch. (11) is solved with respect to $i_{b}$ and the solution

$i_{b}\left(P_{b}\right)=\frac{\eta_{s s}^{\operatorname{sign}\left(P_{b}\right)} V_{o c}-\sqrt{\eta_{s s}^{2 \operatorname{sign}\left(P_{b}\right)} V_{o c}^{2}-4 P_{b} \eta_{s s}^{\operatorname{sign}\left(P_{b}\right)} R_{b}}}{2 \eta_{s s}^{\operatorname{sign}\left(P_{b}\right)} R_{b}}$

is applied to reformulate (9), leading to the differential equation of SoC with respect to $P_{b}$.
3) Inverter, electric motor and transmission: The PS power $P_{r}$ and the SS power $P_{b}$ are merged at the DC link, yielding $P_{p l}$ that is turned into $\mathrm{AC}$ power $P_{i}$ by the bidirectional inverter. By analogy to the AC-DC rectifier, the inverter is also modeled as a constant efficiency factor $\eta_{i}$. The power flow through the DC-link and inverter is described by:

$$
P_{i}=\eta_{i}^{\operatorname{sign}\left(P_{r}+P_{b}\right)}\left(P_{r}+P_{b}\right)=\eta_{i}^{\operatorname{sign}\left(P_{p l}\right)} P_{p l} .
$$

The PMS motor/generator offers bi-directional energy conversion, when it works as a motor, the electric power $P_{i}$ is converted into mechanical power $P_{m}$ for propulsion. Conversely, $P_{m}$ is transformed into $P_{i}$ when it works as a generator. The efficiency map of the PMS motor/generator is shown in Fig. 5, based on which the efficiency of each operating point can be determined by load torque $\tau_{l m}$ and angular speed $\omega_{m}$ of the PMS machine.

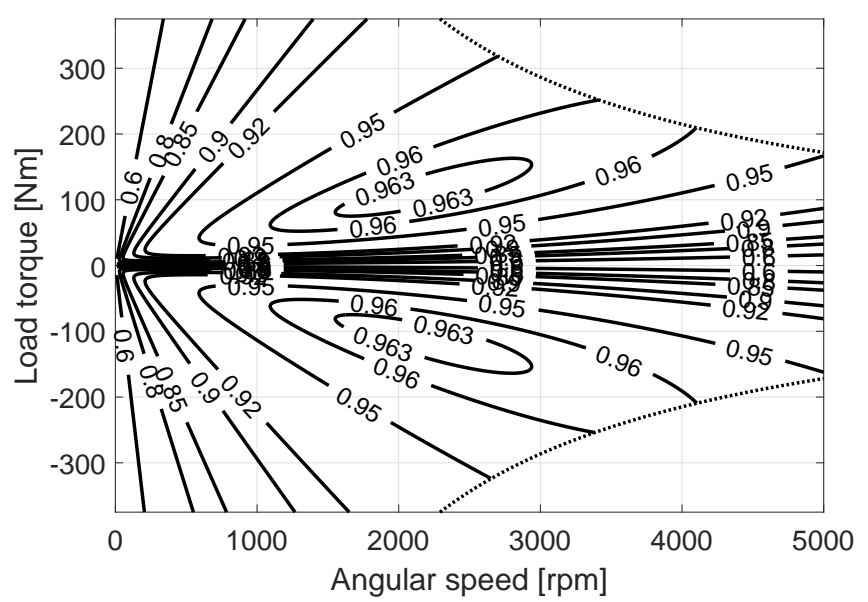

Fig. 5. Efficiency of the reversible PMS machine (generator = positive torque, motor $=$ negative torque).

The PMSM drives the wheels via a transmission system with a fixed ratio $N$, such that

$$
\omega_{m}=N v \text {. }
$$

The bi-directional power flow through the transmission is modeled by:

$$
P_{t}=\eta_{t}^{\operatorname{sign}\left(P_{m}\right)} P_{m}
$$

with $\eta_{t}$ a constant efficiency factor. Combing all three components of the PL branch, the overall efficiency of PL is defined as: $\eta_{p l}=\eta_{i} \eta_{m} \eta_{t}$, which is a function of $v$ and $P_{t}$. It is worth noting that $P_{t}=v F_{v}$ when $F_{v} \geq 0$ and when $F_{v} \leq 0, P_{t}$ is a fraction of $v F_{v}$ because of the mechanical brakes.

\section{B. Engine management optimization}

The fact that the ICE is mechanically decoupled from the vehicle wheels, allows the ICE to be control independently of the vehicle speed. The problem here is to identify the best operating point of the EGU in terms of speed and torque that maximizes branch efficiency for given power demand $P_{r}$ [12], [13]. The locus of such torque-speed operating points is shown on the efficiency map Fig. 4, along which the engine fuel mass rate $m_{f}$ only depends the primary source power 
output $P_{r}$, and in this case the dependency is nearly linear so equation (8) can be converted into:

$$
\frac{d}{d t} m_{f}=q_{f 0}+\frac{P_{r}}{Q_{H V} \alpha_{f}}
$$

$q_{f 0}=0.12 \mathrm{~g} / \mathrm{s}$ is the fuel mass rate to keep the engine idle and $\alpha_{f}=0.34$ is the coefficient of power transformation.

\section{Powertrain power-split optimization (OCP-EM)}

For a given speed profile, $F_{v}$ is uniquely determined by (3). To find the powers $P_{r}, P_{b}$ provided by the primary and secondary sources which minimized fuel consumption, the cost to be minimized is

$$
J=m_{f}(T)
$$

and the state space model is:

$$
\frac{d}{d t}\left(\begin{array}{c}
m_{f} \\
\mathrm{SoC} \\
F_{r} \\
F_{b}
\end{array}\right)=\left(\begin{array}{c}
q_{f 0}+v F_{r} /\left(Q_{H V} \alpha_{f}\right) \\
-i_{b}\left(v F_{b}\right) / Q_{\max } \\
m j_{r} \\
m j_{b}
\end{array}\right)
$$

where $F_{b}, F_{r}$ are the power $P_{b}, P_{r}$ associated forces, $i_{b}\left(v F_{b}\right)$ is given in (12) and jerk decision variables $\mathbf{u}=$ $\left[j_{r}, j_{b}\right]^{T}$ have been introduced for a smoother operation as mentioned previously in Section III.

The following equality and inequality constraints complete the problem:

$$
\begin{array}{r}
v F_{r}+v F_{b} \geq v F_{v} \eta_{p l}^{\operatorname{sign}\left(-F_{v}\right)} \\
-j_{b, \max } \leq j_{b} \leq j_{b, \text { max }} \\
-j_{r, \max } \leq j_{r} \leq j_{r, \max } \\
0 \leq v F_{r} \leq P_{r, \max } \\
\mathrm{SoC}_{\min } \leq \mathrm{SoC} \leq \mathrm{SoC}_{\max } \\
P_{b, \min } \leq v F_{b} \leq P_{b, \text { max }}
\end{array}
$$

The equality constraint (19a) is proposed to reflect the power balance at DC-link:

$$
v F_{r}+v F_{b}=P_{t} \eta_{p l}^{\operatorname{sign}\left(-F_{v}\right)} .
$$

More specifically, when $F_{v} \geq 0, P_{t}=v F_{v}$, and the resulting equality constraint $v F_{r}+v F_{b}=v F_{v} \eta_{p l}^{\operatorname{sign}\left(-F_{v}\right)}$ is naturally equivalent to (19a) because $v F_{r}+v F_{b}$ is minimized to reduce the overall fuel consumption. On the other hand, it is evident that (19a) is also valid when $F_{v}<0$, as $P_{t}>v F_{v}$ in this case. (19b) and (19b) are imposed with $j_{b, \max }=j_{r, \max }=$ $1 \mathrm{~m} / \mathrm{s}^{3}$ for smooth operation analogous to $j_{v}$ introduced in OCP-S. To keep the powertrain components operated inside their admissible range, (19d)-(19f) are needed. In this work, we let $\mathrm{SoC}_{\min }=0.5, \mathrm{SoC}_{\max }=0.8$ to ensure the battery is closely charge sustained throughout the mission. Moreover, $P_{r, \max }=75 \mathrm{~kW}, P_{b \min }=-15 \mathrm{~kW}$ and $P_{b \max }=30 \mathrm{~kW}$, which account for the various losses from the energy sources to the DC-link.
Finally, the problem is completed by the following boundary conditions:

$$
\begin{array}{lr}
\operatorname{SoC}(0)=0.65, & \operatorname{SoC}(T)=0.65, \\
F_{b}(0)=0, & F_{b}(T)=0, \\
F_{r}(0)=0, & F_{r}(T)=0, \\
m_{f}(0)=0 &
\end{array}
$$

where SoC is assumed at the beginning to be at the middle of the allowed $\mathrm{SoC}$ range. To cancel the equivalent fuel caused by the non-zero differences between and initial and terminal conditions of SoC, $F_{b}$ and $F_{r}$, (20a)-(20c) are employed.

To solve OCP-EM, it is necessary to introduce a specific speed profile, which essentially defines $P_{p l}$ to be fulfilled. For the sake of further comparison, we feed the OCP-EM optimal speed profiles produced by OCP-S (see the OCPSplit shown in Fig. 2). For example, the optimal solutions driven by $\rho=0$ and $\rho=1$ are reported in Fig. 6. It is illustrated that following the profiles obtained by OCP$S$ requests very high power withdrawal from both energy sources for very short duration. In contrast with the case $\rho=1$, where both engine and battery provide small amount of power persistently to balance the tyre and aerodynamics drag resistance losses during the cruising period, both energy sources are nearly off to let the vehicle glide down to a lower speed when $\rho=0$. Eventually, less braking power is needed to stop the vehicle, removing the need for using substantial mechanical braking power, which is totally wasted.
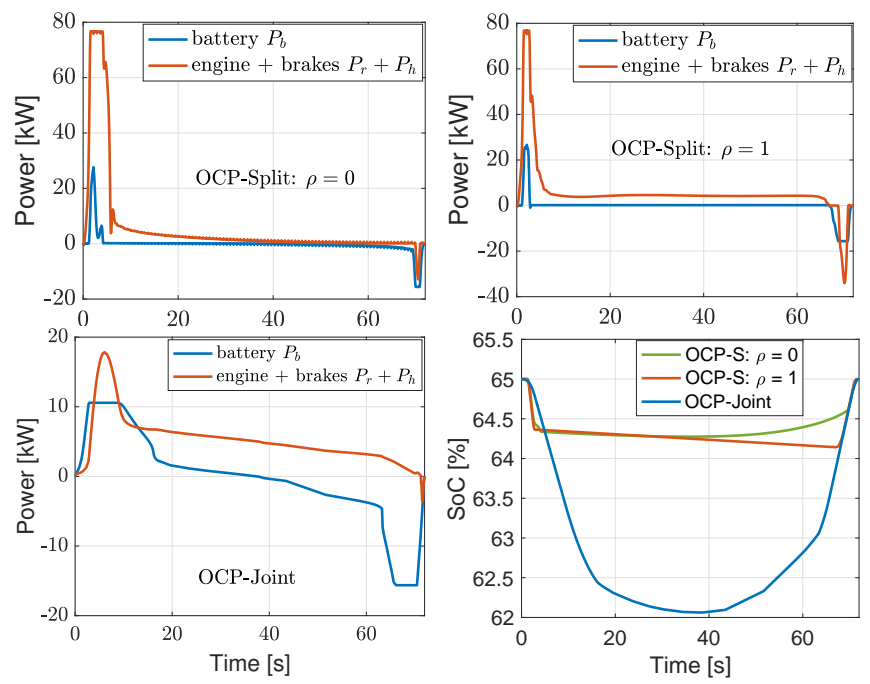

Fig. 6. Power flow and battery SoC of OCP-Joint and OCP-Split with different $\rho=0$ and $\rho=1$, respectively.

\section{JOINT OPTIMIZATION (OCP-JOINT)}

The joint optimization aims to solve OCP-S and OCP-EM simultaneously and globally, considering a single OCP that simply merges OCP-S and OCP-EM formulated previously. The objective is minimize $m_{f}(T)$ by finding the decision variables $\mathbf{u}=\left[j_{v}, j_{b}, j_{r}\right]^{T}$ and the vehicle speed $v$ that is the consequence of $\mathbf{u}$. Next, OCP-Split that consecutively combines OCP-S and OCP-EM is utilized to benchmark the 
OCP-Joint in terms of optimal fuel economy and computational burden.

As demonstrated in Fig. 3 and 6, the optimized vehicle speed of OCP-Joint appears to be different from any solution from OCP-S. The optimal solution of OCP-Joint applies much slower acceleration and deceleration because of the inclusion of transmission losses, by which high power transmission is penalized. As a consequence, the power flow and SoC profile solved by the joint optimization are very different from the results obtained by OCP-Split. In particular, the formulation of OCP-Joint makes it possible to take more advantages of the battery, thus the mechanical brake is minimized during the braking phase.

Overall, the vehicle fuel economy greatly benefits from the joint optimization. As can be seen in Fig. 7, the fuel economy delivered by the OCP-Joint is $4.75 \mathrm{~L} / 100 \mathrm{~km}$, that saves approximately $5.5 \%$ fuel usage as compared to the best behaved OCP-Split parametrized by $\rho=0.5$. The benefit of OCP-Joint increases as $\rho$ diverges from 0.5 , which may be the case closest to the average energy recovery factor solved by the joint optimization among the choices of $\rho$. Noticeably, the greatest disparity appears when $\rho=1$, by which the OCP-Split lags about $13.3 \%$ in terms of fuel economy.

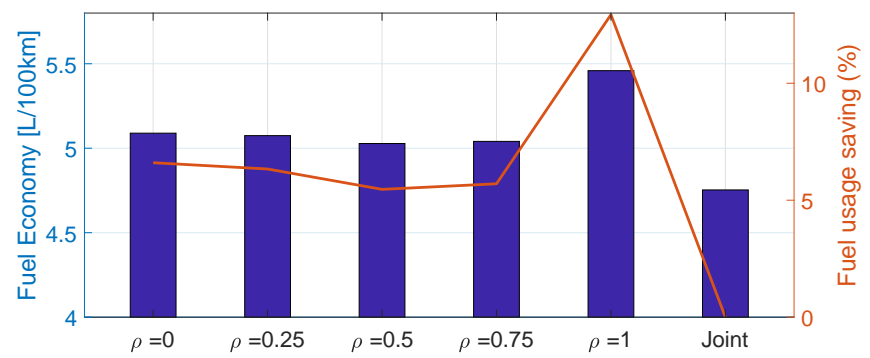

Fig. 7. Comparison of fuel economy delivered by the OCP-Joint and the OCP-Split with different $\rho$.

Table II shows the average computation time required by the OCP-Joint and the OCP-Split, respectively. The requested

TABLE II

AVERAGE COMPUTATION TIME REQUIRED BY THE OCP-SPLIT AND THE OCP-JOINT. THE CPU USED FOR COMPUTATION IS INTEL XEON E5-1650, $3.5 \mathrm{GHz}$.

\begin{tabular}{lc}
\hline \hline & Average running Time [s] \\
\hline OCP-Joint & 34.63 \\
OCP-Split & 15.88 \\
\hline \hline
\end{tabular}

computation time of OCP-Joint is doubled in comparison to the OCP-Split, although it offers overall better performance than the OCP-Split. As the complexity of the problem increases by involving more practical aspects, for example, road gradient and traffic influence, the OCP-Joint may easily become unfeasible, while in that case OCP-Split could be a computationally efficient alternative.

\section{CONCLUDING REMARKS}

The operation of HEV can be optimized in several aspects to reduce fuel consumption. This paper summarizes possible optimization problems that can be formulated in the context of HEVs with the series powertrain architecture. The concerned optimization problems are formulated as OCPs with the aim of solving a practical and global problem with the drive mission defined in terms of traveling time and route characteristics, which are easily available from a navigation system. The two-step optimization scheme that collects two conventional optimization strategies respectively for vehicle speed and energy management, is utilized to benchmark the approach that optimizes both aspects jointly. It is demonstrated that the joint optimization improves the fuel economy by $6 \%-14 \%$ as compared to the two-step counterpart at the expense of nearly doubled computation time.

\section{REFERENCES}

[1] S. E. Li, H. Peng, K. Li, and J. Wang, "Minimum fuel control strategy in automated car-following scenarios," IEEE Transactions On Vehicular Technology, vol. 61, no. 3, pp. 998-1007, 2012.

[2] W. Shabbir and S. A. Evangelou, "Real-time control strategy to maximize hybrid electric vehicle powertrain efficiency," Applied Energy, pp. 512-522, 2014.

[3] T. Liu, X. Hu, S. E. Li, and D. Cao, "Reinforcement learning optimized look-ahead energy management of a parallel hybrid electric vehicle," IEEE Transactions on Mechatronics, vol. 22, no. 4, pp. 1497-1507, 2017.

[4] D. A. Crolla, Q. Ren, S. ElDemerdash, and F. Yu, "Controller design for hybrid vehicles - state of the art review," in IEEE Vehicle Power and Propulsion Conference, sept. 2008, pp. $1-6$.

[5] A. Sciarretta, M. Back, and L. Guzzella, "Optimal control of parallel hybrid electric vehicles," IEEE Transactions on Control Systems Technology, vol. 12, no. 3, pp. 352-363, 2004.

[6] L. Serrao, S. Onori, and G. Rizzoni, "A comparative analysis of energy management strategies for hybrid electric vehicles," Journal of Dynamic Systems, Measurement, and Control, 2011.

[7] C. Musardo, G. Rizzoni, and B. Staccia, "A-ECMS: An adaptive algorithm for hybrid electric vehicle energy management," in Decision and Control, 2005 and 2005 European Control Conference. CDC-ECC '05. 44th IEEE Conference on, Dec. 2005, pp. 1816 - 1823.

[8] L. V. Pérez and E. A. Pilotta, "Optimal power split in a hybrid electric vehicle using direct transcription of an optimal control problem," Math. and Comput. in Simulation, vol. 79, no. 6, pp. 1959-1970, February 2009.

[9] W. Zhou, C. Zhang, J. Li, and H. K. Fathy, "A pseudospectral strategy for optimal power management in series hybrid electric powertrains," IEEE Transactions On Vehicular Technology, vol. 65, no. 6, pp. 4813$4825,2016$.

[10] J. Zhang and T. Shen, "Real-time fuel economy optimization with nonlinear mpc for phevs," IEEE Transactions On Control Systems Technology, vol. 24, no. 6, pp. 2167-2175, 2016.

[11] Y. Zhang, H. Liu, and Q. Guo, "Varying-domain optimal management strategy for parallel hybrid electric vehicles," IEEE Transactions On Vehicular Technology, vol. 63, no. 2, pp. 603-616, 2014.

[12] R. Lot and S. A. Evangelou, "Green driving optimization of a series hybrid electric vehicle," in 52nd IEEE Conference on Decision and Control, 2013, pp. 2200-2207.

[13] B. Chen, S. Evangelou, and R. Lot, "Impact of optimally controlled continuously variable transmission on fuel economy of a series hybrid electric vehicle," in European Control Conference, Limassol, Cyprus, 2018.

[14] A. V. Rao, D. Benson, C. Darby, M. Patterson, C. Francolin, I. Sanders, and G. Huntington, "Gpops: A matlab software for solving multiple-phase optimal control problems using the gauss pseudospectral method," ACM Transactions on Mathematical Software, vol. 37, no. 2,2010

[15] V. Johnson, "Battery performance models in advisor," Journal of Power Source, vol. 110, no. 2, pp. 321-329, 2002. 